\title{
LUT
}

University

\section{Adaptive square-root unscented Kalman filter: An experimental study of hydraulic actuator state estimation}

Mohammadi Asl Reza, Shabbouei Hagh Yashar, Simani Silvio, Handroos Heikki

This is a Author's accepted manuscript (AAM) version of a publication published by Elsevier

in Mechanical Systems and Signal Processing

DOI: $10.1016 /$ j.ymssp.2019.07.021

Copyright of the original publication: () 2019 Elsevier

Please cite the publication as follows:

Mohammadi Asl, R., Shabbouei Hagh, Y., Simani, S., Handroos H. (2019). Adaptive square-root unscented Kalman filter: An experimental study of hydraulic actuator state estimation. Mechanical Systems and Signal Processing, vol. 132. pp. 670-691. DOI: 10.1016/j. ymssp.2019.07.021

This is a parallel published version of an original publication. This version can differ from the original published article. 


\section{Accepted Manuscript}

Adaptive Square-root Unscented Kalman Filter: An experimental study of hydraulic actuator state estimation

Reza Mohammadi Asl, Yashar Shabbouei Hagh, Silvio Simani, Heikki

Handroos

PII:

S0888-3270(19)30451-0

DOI:

https://doi.org/10.1016/j.ymssp.2019.07.021

Reference:

YMSSP 6240

To appear in:

Mechanical Systems and Signal Processing

Received Date:

2 May 2019

Accepted Date:

12 July 2019

Please cite this article as: R.M. Asl, Y.S. Hagh, S. Simani, H. Handroos, Adaptive Square-root Unscented Kalman Filter: An experimental study of hydraulic actuator state estimation, Mechanical Systems and Signal Processing (2019), doi: https://doi.org/10.1016/j.ymssp.2019.07.021

This is a PDF file of an unedited manuscript that has been accepted for publication. As a service to our customers we are providing this early version of the manuscript. The manuscript will undergo copyediting, typesetting, and review of the resulting proof before it is published in its final form. Please note that during the production process errors may be discovered which could affect the content, and all legal disclaimers that apply to the journal pertain. 


\title{
Adaptive Square-root Unscented Kalman Filter: An experimental study of hydraulic actuator state estimation
}

\author{
Reza Mohammadi Asl ${ }^{\mathrm{a}}$, Yashar Shabbouei Hagh ${ }^{\mathrm{a}, *}$, Silvio Simani ${ }^{\mathrm{b}}$, Heikki Handroos $^{\mathrm{a}}$ \\ ${ }^{a}$ Laboratory of Intelligent Machines,Department of Mechanical Engineering, LUT University,Lappeenranta, Finland \\ ${ }^{b}$ Department of Engineering, University of Ferrara, Ferrara, Italy
}

\begin{abstract}
This paper introduces a new adaptive Kalman filter for nonlinear systems. The proposed method is an adaptive version of the square-root unscented Kalman filter (Sr-UKF). The presented adaptive square-root unscented Kalman filter (ASr-UKF) is developed to estimate/detect the states of a nonlinear system while noise statistics that affect system measurement and states are unknown. The filter attempts to adaptively estimate means and covariances of both process and measurement noises and also the states of the system simultaneously. This evaluation of the value of covariances helps the filter to modify itself in order to have more precise estimation. To test the efficiency of the investigated filter, it is applied to different approaches, including state estimation and fault detection. First, the proposed filter is used to predict states of two different nonlinear systems: a robot manipulator and a servo-hydraulic system. Second, the filter is employed to detect a leakage fault in a hydraulic system. All applications are tested under three assumptions: noises with known constant statistics, noises with unknown constant statistics and noises with unknown time-varying statistics. Simulation and experimental results prove the efficiency of the presented filter in comparison with the previous version.

Keywords: Adaptive square-root unscented Kalman filter, State estimation, Fault detection and diagnosis, Robot manipulator, Servo-hydraulic system, Noise mean and covariance estimation
\end{abstract}

\section{Introduction}

Estimation of the internal states of a practical system is an important part of controlling every system. New methods must be developed to estimate the states. Many different reasons can be pointed out to indicate the importance of state estimation: some sensors are too expensive to be implemented and some physical components cannot be measured with sensors. These problems make it reasonable to investigate new methods to estimate or detect the states of the systems. Regarding the purpose of the estimation methods, the definition of state estimation can be given as matching a time series on the propagation of states of the system.

In recent years, different types of filtering approaches, such as model based ones [1, have been developed for different purposes, such as system estimator, and reflection of cyber-attacks in different systems [2, 3, 4]. For instance, a new state estimator has been developed in [5. The proposed algorithm has been designed based on a combination of a pole placement method and linear matrix inequality. The proposed method has been employed as

\footnotetext{
${ }^{*}$ Corresponding author

Email addresses: reza.mohammadi.asl@lut.fi (Reza Mohammadi Asl), yashar.shabbouei.hagh@lut.fi (Yashar Shabbouei Hagh), silvio.simani@unife.it (Silvio Simani), heikki.handroos@lut.fi (Heikki Handroos)
} 
a larger scheme to control the decentralized secondary voltage. In other research, a new method has been proposed to estimate the states of noisy systems [6]. The method has been developed to estimate the states in the presence of sparse sensor integrity attacks. The presented method was a combination of robust control and fault detection and isolation concepts. Various types of state estimators have been developed previously, such as particle filtering [7] and Kalman filtering [8]. For example, a new sequential Monte Carlo approach as a particle filter has been developed in 9. The filter uses a nonlinear model for measurement. The purpose of the particle filter is to have a filter with an acceptable performance in the presence of non-Gaussian noise.

For linear systems, although different methods with acceptable results have been designed, it has been proved that the Kalman filter has the best estimation of the real system. The Kalman filter is a well-known method introduced by Rudolf Kalman. The filter has been developed to estimate the states of the system based on some measurements of the system in each time step [10. Regarding the performance of the Kalman filter, it has a wide usage in system estimation procedures. For example, a combination of Kalman filter and neural network has been introduced for detecting data fusion in [11. The method has been developed based on different assumptions: known system model, describing the mathematical relationship between sampling rates. As can be drawn from the wide usage of the filter, it has a good performance on linear systems, but it requires some modifications to estimate nonlinear systems. Owing to this fact, many researchers have developed state estimation methods for nonlinear systems. For example, an estimation has been investigated based on Markovian channels in [12. The method has been developed to solve the estimation problem in networked systems. Different versions of Kalman filter have been presented for nonlinear systems. The most common and conventional nonlinear versions are the cubature Kalman filter [13], extended Kalman filter (EKF) [14, and unscented Kalman filter (UKF) [15. These filters have various applications, such as parameter estimation in diesel-engine SCR system [16, but state estimation is the most common application among. For instance, the UKF has been used as part of a controlling system for a robotic manipulator in [17. The filter has been combined with an evolutionary algorithm to estimate the states of the robotic system. As another example, in [18] computational load has been decreased by implementation of a new dual time-scale UKF which also assured the implementation performance. The proposed filter is trying to estimate the hydrothermal aging factor in a diesel engine.

The previously introduced methods have some disadvantages that can be summarized as follows. In almost all of the literature cited previously, it has been assumed that noise mean and power, both for process and measurement noises, are known previously. In other words, a priori knowledge about noise statistics is assumed. This a priori knowledge is almost always difficult and in some cases impossible to pre-define. The other drawback of the approaches in the literature is assuming fixed noise statistics. The papers have assumed that noise mean and covariance are constant over time and no change in their values are considered. Any mismatch between the real noises and the ones that are assumed, and any changes in the mean and covariance matrix of the noise over time, may lead to inaccurate estimations and even in some cases result in divergence. In some newer researches, some adaptive Kalman filters have been introduces which are tried to solve the mismatching problem between real and supposed noise covariances[19]. For instance, a new weighted adaptive version of unscented Kalman filter has been developed and introduced in 20]. Although the presented method has solved mismatching problem by the estimation of noise covariance and mean, positive semi-definiteness of the states covariances has not been guaranteed. In another research, an adaptive 

mismatch 21.

To tackle the problems of the previous methods, it is necessary to design new strategies that can solve some or all of them. Regarding this topic, a new adaptive square-root unscented Kalman filter (ASr-UKF) is presented. As indicated in [22, 23] the Sr-UKF not only solves the drawbacks of the EKF and UKF, but also guarantees the mentioned problems. The adaptive filter does not need any prior knowledge about the measurement and process noises, and not only estimates the states of the nonlinear system but is also able to estimate the covariances of noises. Regarding this ability, the filter can predict the states of the system during any change in the statistics of noises. In the procedure of designing this filter, no extra assumptions are considered. To show the proficiency of the presented method, two different applications of the filter are discussed. As for state estimation, it is applied to two different systems: a robotic manipulator and a servo-hydraulic system. The results of simulations are provided.

On the other hand, the Kalman filter as a model-based fault detection method [24, 25] is in wide usage for fault detection and diagnosis (FDD) in different types of systems [26, 27]. For instance, a combination of the Kalman filter and fault factor methods has been introduced for fault detection and isolation (FDI) in 28]. The proposed method has been an interesting research topic in recent years. For example, an EKF has been employed to detect leakage faults in 29]. The proposed method has been designed to detect the fault in the presence of uncertainties in the system model as an unknown external loading. To evaluate the performance of the presented filter in this research, it also been tested as an FDD module. The hydraulic actuator leakage fault is studied to show the performance of the proposed filter as an FDD method.

The rest of this paper is organized as follows. In Section 2 the principles of the proposed ASr-UKF are described. First, the conventional square-root unscented Kalman filter (Sr-UKF) is discussed and then the adaptive filter is presented, which deals with the drawback of the Sr-UKF. The simulation and experimental result parts are addressed in Section 3 and consists of three subsections. First, the application of the filter as a state estimator is described, and it is examined over two different case studies, namely a six-degree-of-freedom (6-DOF) robot manipulator and a servo-hydraulic system. The dynamics of these two systems are addressed and then two scenarios are simulated to demonstrate the efficiency of the ASr-UKF; constant and time-varying noise statistics are considered. For the second application, the proficiency of the ASr-UKF is simulated on the leakage FDD of a hydraulic system. The paper is concluded in Section 4

\section{Adaptive Square-root Unscented Kalman Filter}

As mentioned previously, regarding nonlinear systems, different approaches have been introduced to approximate the nonlinearities of the system's dynamics, such as EKF and UKF. The drawbacks of these methods are discussed and addressed. In this section, the principles of the Sr-UKF are proposed first; then, an adaptive version of the filter is given, which outperforms the conventional Sr-UKF in the case of unknown or time-varying noise distributions. 
Consider the following nonlinear system:

$$
\begin{aligned}
& \dot{x}(t)=f(x(t), u(t))+w(t) \\
& y(t)=g(x(t))+v(t)
\end{aligned}
$$

where $x \in R^{n}$ represents the state vector, and $y \in R^{m}$ and $u \in R^{L}$ represent the outputs and inputs, respectively. The nonlinear dynamics and measurements of the system are represented by the functions $f$ and $g$. The system is affected by Gaussian white process and measurement noise distributions, indicated as $w \sim N(0, Q)$ and $v \sim N(0, R)$, respectively. It is assumed that these noises have zero mean and covariance matrices $Q$ and $R$.

Note that, if $T_{s}$ is the sampling period, the variable values are considered at each sampling time $k . T_{s}$; however, to simplify notation, in the rest of the paper, $k$ will be used instead of $k \cdot T_{s}$.

The first step of the algorithm is the initialization. In this phase, the initial values of states and the square-root of the error covariance matrix, $\left(S_{k}\right)$, are considered as follows:

$$
\begin{aligned}
& \hat{x}_{0}=E\left[x_{0}\right] \\
& S_{0}=C H\left(E\left[\left(x_{0}-\hat{x}_{0}\right)\left(x_{0}-\hat{x}_{0}\right)^{T}\right]\right)
\end{aligned}
$$

the function $C H$ represents the Cholesky factor of $E\left[\left(x_{0}-\hat{x}_{0}\right)\left(x_{0}-\hat{x}_{0}\right)^{T}\right]$.

Next, using the a priori state $\left(\hat{x}_{k-1 \mid k-1}\right)$ and Cholesky factor $\left(S_{k-1 \mid k-1}\right)$, the sigma points $\left(\mathcal{X}_{k-1 \mid k-1}^{(i)}\right)$ are calculated as

$$
\mathcal{X}_{k-1 \mid k-1}^{(i)}=\left[\hat{x}_{k-1 \mid k-1}, \hat{x}_{k-1 \mid k-1}+\sqrt{L+\lambda} S_{k-1 \mid k-1}, \hat{x}_{k-1 \mid k-1}-\sqrt{L+\lambda} S_{k-1 \mid k-1}\right]
$$

Here, $i=0,1, \ldots, 2 L$ where $L$ is the number of states and $\lambda$ is a scaling parameter defined as $\lambda=L\left(\alpha^{2}-1\right)$. In this article, $\alpha$ is a weighting factor, which is set between $10^{-4}$ and 1 . The calculated sigma points are now propagated through the nonlinear dynamics function $f$

$$
\gamma_{k \mid k-1}^{(i)}=f\left(\mathcal{X}_{k-1 \mid k-1}^{(i)}, u_{k-1 \mid k-1}\right), \quad i=0,1, \ldots, 2 L
$$

The a posteriori mean and time update of the Cholesky factor, $S_{k \mid k-1}$, are thus calculated:

$$
\begin{aligned}
\hat{x}_{k \mid k-1} & =\sum_{i=0}^{2 n} w_{i}^{(m)} \gamma_{k \mid k-1}^{(i)} \\
S_{k \mid k-1} & =Q R\left(\left[\sqrt{w_{i}^{(c)}}\left(\gamma_{k \mid k-1}^{(i)}-\hat{x}_{k \mid k-1}\right) \sqrt{Q}\right]\right) \\
S_{k \mid k-1} & =C U\left(S_{k \mid k-1}, \gamma_{k \mid k-1}^{(0)}-\hat{x}_{k \mid k-1}, w_{0}^{(c)}\right)
\end{aligned}
$$

where the function $Q R(A)$ returns the matrix $S$ that satisfies the equation $A=Z \times S$. In this equation, $S$ is an upper triangular matrix and $Z$ is a unitary matrix. The function $C U$ represents the rank-one update of the Cholesky factorization. The predefined weights $w_{i}^{(m)}$ and $w_{i}^{(c)}$ used in $(5)$ are calculated by

$$
\begin{aligned}
& w_{0}^{(m)}=\frac{\lambda}{L+\lambda} \\
& w_{0}^{(c)}=\frac{\lambda}{L+\lambda}+\left(1-\alpha^{2}+\beta\right) \\
& w_{i}^{(m)}=w_{i}^{(c)}=\frac{1}{2(L+\lambda)} ; \quad i=1, \ldots, 2 L
\end{aligned}
$$


in which the secondary scaling parameter $\beta$ is usually set to 2 for a Gaussian distribution [30. In (5) QR represents the QR decomposition. On the other hand, because the weight, $w_{0}^{(c)}$, might be negative, the subsequent Cholesky update, known as downdate, is necessary and is calculated by the choleupdate function, defined as follows. Considering $A$, for instance, as the original Cholesky factor of the matrix $P, A=\operatorname{chol}\{P\}$, choleupdate $\{A, B, c\}$ returns the Cholesky factor of the matrix $P+c B B^{T}$. It should be mentioned that the $C H, Q R$, and $C U$ functions denote the corresponding MATLAB ${ }^{\circledR}$ chol, qr, and cholupdate functions. Therefore, the output should be updated. To this end, the sigma points need to be updated:

$$
\mathcal{X}_{k \mid k-1}^{(i)}=\left[\begin{array}{lll}
\hat{x}_{k \mid k-1} & \hat{x}_{k \mid k-1}+\sqrt{L+\lambda} S_{k \mid k-1} & \hat{x}_{k \mid k-1}-\sqrt{L+\lambda} S_{k \mid k-1}
\end{array}\right]
$$

using the nonlinear measurement function, $g$. Therefore, each column of the updated sigma points are propagated as

$$
\mathcal{Y}_{k \mid k-1}^{(i)}=g\left(\mathcal{X}_{k \mid k-1}^{(i)}\right), \quad i=0,1, \ldots, 2 L
$$

then the Cholesky factors of the observation-error covariance matrices, $S_{k}^{y y}$ and $P_{k}^{x y}$, are calculated as

$$
\begin{aligned}
& \hat{y}_{k \mid k-1}=\sum_{i=0}^{2 n} w_{i}^{(m)} \mathcal{Y}_{k \mid k-1}^{(i)} \\
& S_{k}^{y y}=Q R\left(\left[\sqrt{w_{i}^{(c)}}\left(\mathcal{Y}_{k \mid k-1}^{(i)}-\hat{y}_{k \mid k-1}\right) \sqrt{R}\right]\right) \\
& S_{k}^{y y}=C U\left(S_{k}^{y y}, \mathcal{Y}_{k \mid k-1}^{(0)}-\hat{y}_{k \mid k-1}, w_{0}^{(c)}\right) \\
& P_{k}^{x y}=\sum_{i=0}^{2 n}\left\{w_{i}^{(c)}\left(\gamma_{k \mid k-1}^{(i)}-\hat{x}_{k \mid k-1}\right)\left(\mathcal{Y}_{k \mid k-1}^{(i)}-\hat{y}_{k \mid k-1}\right)^{T}\right\}
\end{aligned}
$$

therefore, the Kalman filter gain will be calculated using these matrices

$$
K=P_{k}^{x y} /\left(S_{k}^{y y}\right)^{T} / S_{k}^{y y}
$$

The a posteriori estimated state and Cholesky factor $S_{k \mid k}$ can be computed using the Kalman gain

$$
\begin{aligned}
& \hat{x}_{k \mid k}=\hat{x}_{k \mid k-1}+K_{k}\left(y_{k}-\hat{y}_{k \mid k-1}\right) \\
& U=K_{k} S_{k}^{y y} \\
& S_{k \mid k}=C U\left(S_{k \mid k-1}, U,-1\right)
\end{aligned}
$$

this concludes the Sr-UKF algorithm.

Reconsidering the relations of $S_{k \mid k-1}$ and $S_{k}^{y y}$ in (5) and (9), respectively, it can be concluded that the process and the measurement covariance matrices, $Q$ and $R$, have a critical effect on their values. Therefore, it is required that the $Q$ and $R$ matrices be known exactly. Hence, any mismatch between real noises that affect the system and those that are assumed in Sr-UKF can reduce the performance of the algorithm, which can also diverge. An adaptive Sr-UKF (ASr-UKF) is thus proposed in this paper, which solves the problem of the Sr-UKF by estimating the process and measurement noise covariances.

Consider the nonlinear dynamic model of (1). Assume that process and measurement noises are defined as $w \sim(q, Q)$ and $v \sim(r, R)$. To estimate the noise covariance and mean values, a posteriori density function is 
assumed in the following form, which should be maximized:

$$
J^{*}=p[X(k), Q, R, q, r \mid Y(k)]=\frac{p[Y(k) \mid X(k), Q, R, q, r] p[X(k), Q, R, q, r]}{p[Y(k)]}
$$

where $X(k)$ is the state vector $\left[x_{0}, x_{1}, \ldots, x_{k}\right]$ and $Y(k)$ is the measurement vector $\left[y_{0}, y_{1}, \ldots, y_{k}\right]$. As $p[Y(k)]$ is not involved in the optimization problem, therefore the $J$ function can be rewritten as the following unconditional density function:

$$
J=p[Y(k) \mid X(k), Q, R, q, r] \times p[X(k) \mid Q, R, q, r] \times p[Q, R, q, r]
$$

In this equation, the term $p[Q, R, q, r]$ is assumed to have a constant value because it can be acquired based on a priori information [31. It is also assumed that the following assumptions are valid, which indicates that noise distributions are not correlated and cross-correlated,

$$
\begin{array}{lc}
\operatorname{Cov}\left[w_{i}, w_{k}\right]=0, & i \neq k \\
\operatorname{Cov}\left[v_{i}, v_{k}\right]=0, & i \neq k \\
\operatorname{Cov}\left[v_{i}, w_{k}\right]=0, & i \neq k
\end{array}
$$

which make it possible to use the multiplication theorem of conditional probabilities, which leads to the following expression:

$$
\begin{aligned}
p\left[X_{k} \mid Q, R, q, r\right]= & p\left[x_{0}\right] \prod_{j=1}^{k} p\left[x_{j} \mid x_{j-1}, q, Q\right] \\
= & \frac{1}{(2 \pi)^{n / 2}\left|P_{0}\right|^{1 / 2}} \exp \left\{-\frac{1}{2}\left\|x_{0}-\hat{x}_{0}\right\|_{P_{0}^{-1}}^{2}\right\} \\
& \times \prod_{j=1}^{k} \frac{1}{(2 \pi)^{n / 2}|Q|^{1 / 2}} \exp \left\{-\frac{1}{2}\left\|x_{j}-f_{j-1}\left(x_{j-1}\right)-q\right\|_{Q^{-1}}^{2}\right\} \\
= & \frac{1}{2 \pi^{n(k+1) / 2}\left|P_{0}\right|^{-1 / 2}|Q|^{-k / 2}} \\
& \times \exp \left\{-\frac{1}{2}\left[\left\|x_{0}-\hat{x}_{0}\right\|_{P_{0}^{-1}}^{2}+\sum_{j=1}^{k}\left\|x_{j}-f_{j-1}\left(x_{j-1}\right)-q\right\|_{Q^{-1}}^{2}\right]\right\} \\
p\left[Y_{k} \mid X_{k}, q, Q, r, R\right]= & \prod_{j=1}^{k} p\left[y_{j} \mid x_{j}, r, R\right] \\
= & \prod_{j=1}^{k} \frac{1}{(2 \pi)^{m / 2}|R|^{1 / 2}} \exp \left\{-\frac{1}{2}\left\|y_{j}-g_{j}\left(x_{j}\right)-r\right\|_{R^{-1}}^{2}\right\} \\
= & \frac{1}{2 \pi^{m k / 2}}|R|^{-k / 2} \exp \left\{-\frac{1}{2} \sum_{j=1}^{k}\left\|y_{j}-g_{j}\left(x_{j}\right)-r\right\|_{R^{-1}}^{2}\right\}
\end{aligned}
$$

where $n$ and $m$ represents the process and the measurement dimension, respectively. The notation $|A|$ indicates the determinant of a square matrix $A$, whereas $\|u\|_{A}^{2}=u^{T} A u$ is the quadratic form.

By considering the relations in (14), the estimation problem can be formulated as an optimization problem of the cost function $J$ : 


$$
\begin{aligned}
J & =\frac{1}{2 \pi^{n(k+1) / 2}} \frac{1}{2 \pi^{m k / 2}}\left|P_{0}\right|^{-1 / 2}|Q|^{-k / 2}|R|^{-k / 2} p[q, Q, r, R] \\
& \times \exp \left\{-\frac{1}{2}\left[\left\|x_{0}-\hat{x}_{0}\right\|_{P_{0}^{-1}}^{2}+\sum_{j=1}^{k}\left\|x_{j}-f_{j-1}\left(x_{j-1}\right)-q\right\|_{Q^{-1}}^{2}+\sum_{j=1}^{k}\left\|y_{j}-g_{j}\left(x_{j}\right)-r\right\|_{R^{-1}}^{2}\right]\right\} \\
& =C|Q|^{-k / 2}|R|^{-k / 2} \exp \left\{-\frac{1}{2}\left[\sum_{j=1}^{k}\left\|x_{j}-f_{j-1}\left(x_{j-1}\right)-q\right\|_{Q^{-1}}^{2}+\sum_{j=1}^{k}\left\|y_{j}-g_{j}\left(x_{j}\right)-r\right\|_{R^{-1}}^{2}\right]\right\}
\end{aligned}
$$

where

$$
C=\frac{1}{2 \pi^{n(k+1) / 2}} \frac{1}{2 \pi^{m k / 2}}\left|P_{0}\right|^{-1 / 2} \cdot p[Q, R] \cdot \exp \left\{\frac{-1}{2}|| x_{0}-\hat{x}_{0} \|_{P_{0}^{-1}}^{2}\right\}
$$

is a constant value.

Now, after computing the derivative of $J$ with respect to $r, q, R$, and $Q$, the noise mean and covariance values can be calculated as

$$
\begin{aligned}
& \hat{Q}_{k}=\frac{1}{k} \sum_{j=1}^{k}\left\{\left[\hat{x}_{j}-f_{j-1}\left(\hat{x}_{j-1}\right)-q\right]\left[\hat{x}_{j}-f_{j-1}\left(\hat{x}_{j-1}\right)-q\right]^{T}\right\} \\
& \hat{q}_{k}=\frac{1}{k} \sum_{j=1}^{k}\left[\hat{x}_{j}-f_{j-1}\left(\hat{x}_{j-1}\right)\right] \\
& \hat{R}_{k}=\frac{1}{k} \sum_{j=1}^{k}\left\{\left[y_{j}-g_{j}\left(\hat{x}_{j \mid j-1}\right)-r\right]\left[y_{j}-g_{j}\left(\hat{x}_{j \mid j-1}\right)-r\right]^{T}\right\} \\
& \hat{r}_{k}=\frac{1}{k} \sum_{j=1}^{k}\left[y_{j}-g_{j}\left(\hat{x}_{j \mid j-1}\right)\right]
\end{aligned}
$$

where the terms $f_{j-1}\left(\hat{x}_{j-1}\right)$ and $g_{j}\left(\hat{x}_{j \mid j-1}\right)$ can be evaluated from the Sr-UKF algorithm as follows:

$$
\begin{aligned}
f_{j-1}\left(\hat{x}_{j-1}\right) & =\sum_{i=0}^{2 n} w_{i}^{(m)} f\left(\mathcal{X}_{j-1 \mid j-1}^{(i)}, u_{k-1}\right) \\
g_{j}\left(\hat{x}_{j \mid j-1}\right) & =\sum_{i=0}^{2 n} w_{i}^{(m)} g\left(\mathcal{X}_{j \mid j-1}^{(i)}\right)
\end{aligned}
$$

The ASr-UKF estimation scheme is represented by Algorithm 1 .

In Algorithm 1, two innovation terms are used. The term $\xi_{k}$ is a parameter to increase the performance of the estimator and $\Gamma_{k}$ is the forgetting factor 32. The subtractions in the $\hat{Q}_{k}$ and $\hat{R}_{k}$ formulas may lead to negative matrices. The following relations can be used for deriving positive-definite matrices:

$$
\begin{aligned}
& \hat{R}_{k}=\hat{R}_{k-1}+\Gamma_{k}\left(\sum_{i=0}^{2 n}\left\{w_{i}^{(c)}\left(\mathcal{Y}_{k \mid k-1}^{(i)}-\hat{y}_{k \mid k-1}\right)\left(\mathcal{Y}_{k \mid k-1}^{(i)}-\hat{y}_{k \mid k-1}\right)^{T}\right\}\right) \\
& \hat{Q}_{k}=\hat{Q}_{k-1}+\Gamma_{k}\left(\sum_{i=0}^{2 n}\left\{w_{i}^{(c)}\left(\gamma_{k \mid k-1}^{(i)}-\hat{x}_{k \mid k-1}\right)\left(\gamma_{k \mid k-1}^{(i)}-\hat{x}_{k \mid k-1}\right)^{T}\right\}\right)
\end{aligned}
$$

\section{Simulations and Experimental Results}

This section summarizes the simulation results achieved from the application of the proposed ASr-UKF filter to 


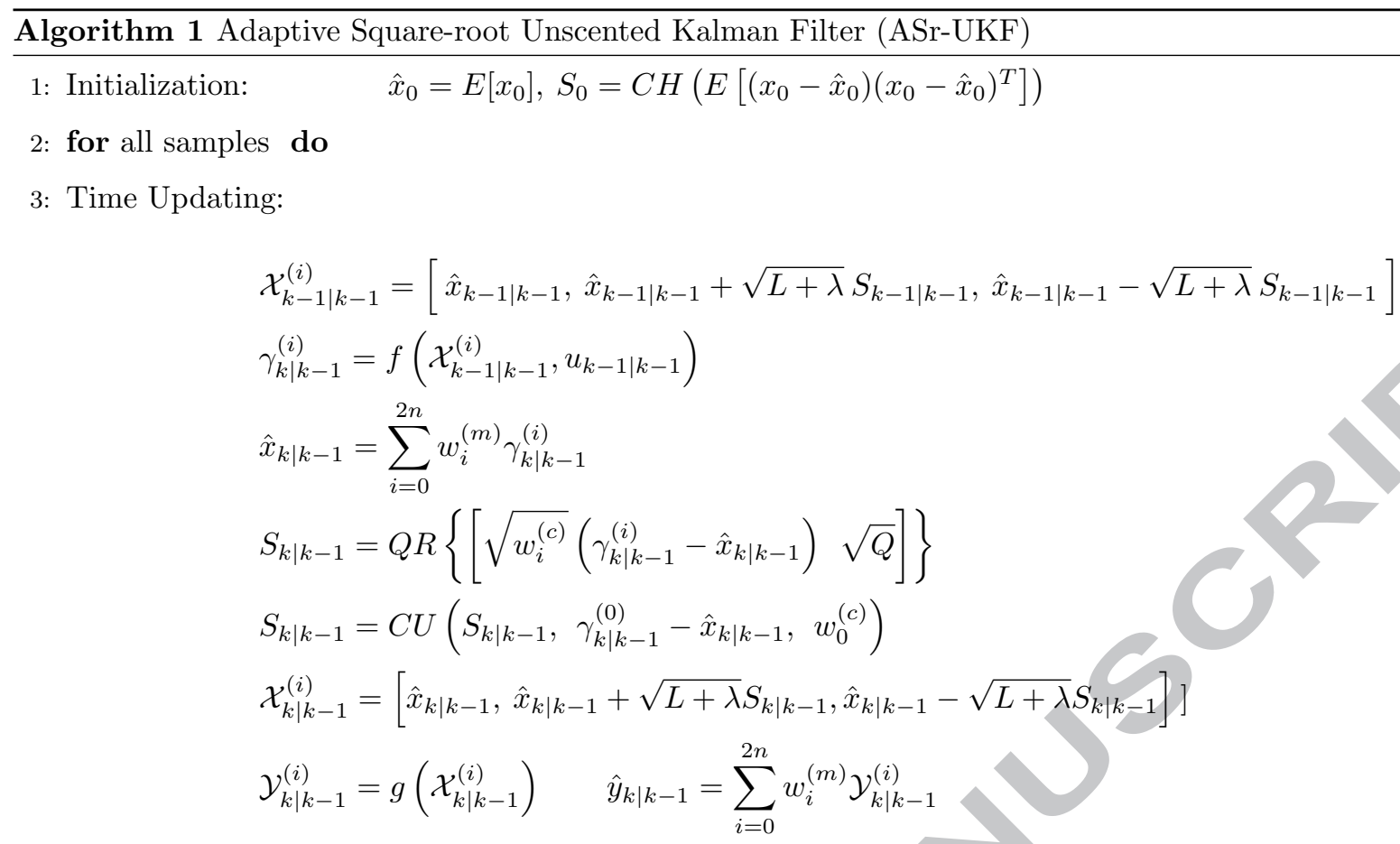

4: Measurement Update:

$$
\begin{aligned}
& S_{k}^{y y}=Q R\left\{\left[\sqrt{w_{i}^{(c)}}\left(\mathcal{Y}_{k \mid k-1}^{(i)}-\hat{y}_{k \mid k-1}\right) \sqrt{R}\right]\right\} \\
& S_{k}^{y y}=C U\left(S_{k}^{y y}, \mathcal{Y}_{k \mid k-1}^{(0)}-\hat{y}_{k \mid k-1}, w_{0}^{(c)}\right) \\
& P_{k}^{x y}=\sum_{i=0}^{2 n}\left\{w_{i}^{(c)}\left(\gamma_{k \mid k-1}^{(i)}-\hat{x}_{k \mid k-1}\right)\left(\mathcal{Y}_{k \mid k-1}^{(i)}-\hat{y}_{k \mid k-1}\right)^{T}\right\}
\end{aligned}
$$

5: States Enhancement:

$$
\begin{aligned}
& K=P_{k}^{x y} /\left(S_{k}^{y y}\right)^{T} / S_{k}^{y y} \\
& \hat{x}_{k \mid k}=\hat{x}_{k \mid k-1}+K_{k}\left(y_{k}-\hat{y}_{k \mid k-1}\right) \\
& U=K_{k} S_{k}^{y y}, \quad S_{k \mid k}=\text { cholupdate }\left\{S_{k \mid k-1}, U,-1\right\}
\end{aligned}
$$

6: Noise Estimation:

$$
\begin{aligned}
& \xi_{k}=y_{k}-\hat{y}_{k \mid k-1}-\hat{r}_{k} \quad \Gamma_{k}=\frac{1-\varrho}{1-\varrho^{k}} \quad 0<\varrho<1 \\
& \hat{R}_{k}=\left(1-\Gamma_{k}\right) \hat{R}_{k-1}+\Gamma_{k}\left[\xi_{k} \xi_{k}^{T}-\sum_{i=0}^{2 n}\left\{w_{i}^{(c)}\left(\mathcal{Y}_{k \mid k-1}^{(i)}-\hat{y}_{k \mid k-1}\right)\left(\mathcal{Y}_{k \mid k-1}^{(i)}-\hat{y}_{k \mid k-1}\right)^{T}\right\}\right] \\
& \hat{Q}_{K}=\left(1-\Gamma_{k}\right) \hat{Q}_{k-1}+\Gamma_{k}\left[K_{k} \xi_{k} \xi_{k}^{T} K_{k}^{T}+S_{k \mid k}-\sum_{i=0}^{2 n}\left\{w_{i}^{(c)}\left(\gamma_{k \mid k-1}^{(i)}-\hat{x}_{k \mid k-1}\right)\left(\gamma_{k \mid k-1}^{(i)}-\hat{x}_{k \mid k-1}\right)^{T}\right\}\right] \\
& \hat{r}_{k}=\left(1-\Gamma_{k}\right) \hat{r}_{k-1}+\Gamma_{k}\left[y_{k}-\sum_{i=0}^{2 n} w_{i}^{(m)} g\left(\mathcal{X}_{k \mid k-1}^{(i)}\right)\right] \\
& \hat{q}_{k}=\left(1-\Gamma_{k}\right) \hat{q}_{k-1}+\Gamma_{k}\left[\hat{x}_{k}-\sum_{i=0}^{2 n} w_{i}^{(m)} f\left(\mathcal{X}_{k-1}^{(i)}, u_{k}\right)\right]
\end{aligned}
$$



(a) First link's position
(b) Estimation error of the first link's position
(c) Second link's position
(d) Estimation error of the second link's position
(e) Third link's position
(f) Estimation error of the third link's position

Figure 1: Comparison of estimated states and estimated errors of the robotic manipulator: components of the robot's position affected by noises with known constant statistics (True: —, ASr-UKF: —, Sr-UKF: —, UKF: —, EKF: - ).

system, three different scenarios are proposed. In the first situation, the filter attempts to estimate the states of the system in the presence of a known noise with constant statistics, to evaluate the performance of the filter in the same condition with same condition with previously introduced filter, whereas the second considers an unknown noise with constant statistics. In the last scenario filter tries to estimates states in presence of an unknown noise with time-varying statistics. The results of all simulations are also presented. Finally, an FDD application has also been considered. In particular, the proposed method is applied for the detection of a leakage in a servo-hydraulic system.

\subsection{Robotic manipulator}

A 6-DOF robotic manipulator is chosen as the second case study. The simulations are performed by fixing the

\subsubsection{Noise with unknown constant statistics}

As mentioned previously, in the first simulation, it is assumed that the robotic manipulator is affected by a noise with constant statistics. For this purpose, the process and measurement noises, which are presented as $w(t)$ and $v(t)$ in (1), are assumed with noise processes with zero mean. The covariance of process noise and measurement 
Table 1: Percent normalized mean square error of estimation of the robotic manipulator affected by noises with known constant statistics

\begin{tabular}{|c|c|c|c|c|c|c|}
\hline Vethod & $\theta_{1}$ & $\theta_{2}$ & $\theta_{3}$ & $\dot{\theta}_{1}$ & $\dot{\theta}_{2}$ & $\dot{\theta}_{3}$ \\
\hline ASr-UKF & 2.91 & 0.58 & 0.41 & 2.32 & 1.96 & 5.16 \\
\hline Sr-UKF & 2.37 & 0.47 & 0.41 & 2.55 & 1.69 & 4.19 \\
\hline AUKF & 2.98 & 0.69 & 0.41 & 2.46 & 2.42 & 5.97 \\
\hline UKF & 2.38 & 0.46 & 0.41 & 2.38 & 1.71 & 4.98 \\
\hline AEKF & 3.15 & 0.67 & 0.54 & 2.41 & 2.58 & 5.89 \\
\hline EKF & 4.63 & 0.81 & 0.47 & 2.66 & 2.58 & 6.16 \\
\hline
\end{tabular}
(a) First link's position
(c) Second link's position
(e) Third link's position

(b) Estimation error of the first link's position

(d) Estimation error of the second link's position

(f) Estimation error of third link's position.

Figure 2: Comparison of estimated states and estimated errors of the robotic manipulator: components of the robot's position affected by noises with constant statistics (True: - ASr-UKF: 一, Sr-UKF: - , UKF: - , EKF: 一).
(a) First link's velocity
(b) Estimation error of the first link's velocity
(c) Second link's velocity
(d) Estimation error of second link's velocity
(e) Third link's velocity
(f) Estimation error of the third link's velocity

Figure 3: Comparison of estimated states and estimated errors of the robotic manipulator: components of the robot's velocity affected by noises with constant statistics (True: $\_$, ASr-UKF: —, Sr-UKF: —, UKF: —, EKF: —).

noise are $Q=10^{-6} I_{6 \times 6}$ and $R=10^{-4} I_{6 \times 6}$, respectively. To highlight the advantages of the proposed filter, it is assumed that there is no a priori knowledge about the statistics of the noises. Based on this assumption, the initial values for the noise statistics are fixed to $\hat{Q}=10^{-20} I_{6 \times 6}$ and $\hat{R}=10^{-20} I_{6 \times 6}$, which are different from the actual noise covariances. The starting values for the robotic model and the proposed filter, ASr-UKF, are initialized as $X_{0}=[1,0.5,1,2,-1,-2]^{T}$ and randomly selected $\hat{X}_{0}=[4.3,2.62,7,6.65,3.5,8.75]^{T}$, respectively. Considering these assumptions, the results of the simulation are reported in Figs. 2 and 3 A comparison is made with traditional filters, Sr-UKF, UKF, EKF, AUKF, and AEKF. As can be seen in Figs. 2 and 3 the new introduced filter has a better performance in comparison with the conventional filters. To provide a further performance metric, the percent normalized mean square errors of the estimation processes are given in Table 2 In the rest of simulations, the application of the proposed ASr-UKF have close results with AUKF and AEKF. Because of that and to have clear figures, the results for AUKF and AEKF are not presented in figures and the comparative results have just presented in tables.

In Figs. 2 and 3 , it is assumed that $\hat{X}_{0}=[4.3,2.62,7,6.65,3.5,8.75]^{T}$. This initial condition is chosen randomly. To evaluate the robustness of the proposed filter with respect to the estimated initial values, different simulations 
Table 2: Percent normalized mean square error of estimation of the robotic manipulator affected by noises with constant statistics

\begin{tabular}{|c|c|c|c|c|c|c|}
\hline Method & $\theta_{1}$ & $\theta_{2}$ & $\theta_{3}$ & $\dot{\theta}_{1}$ & $\dot{\theta}_{2}$ & $\dot{\theta}_{3}$ \\
\hline ASr-UKF & 2.37 & 0.48 & 0.41 & 2.48 & 1.79 & 4.80 \\
\hline Sr-UKF & 88.5 & 11.89 & 20.86 & 8.59 & 12.67 & 17.17 \\
\hline UKF & 93.23 & 33.17 & 32.21 & 7.76 & 10.94 & 16.45 \\
\hline AUKF & 2.39 & 0.48 & 0.39 & 2.47 & 1.82 & 4.96 \\
\hline EKF & 78.12 & 21.00 & 11.49 & 10.32 & 16.95 & 20.57 \\
\hline AEKF & 2.57 & 0.49 & 0.41 & 2.47 & 1.80 & 4.80 \\
\hline
\end{tabular}

with different initial values are performed. The results of the percent normalized mean square error are presented in Table 3. As can be seen, the performance of the proposed filter is notable in different initial conditions. As for $\hat{X}_{0}=[4.3,2.62,7,6.65,3.5,8.75]^{T}$, which has high percent mean square error compared with other initial conditions, it was demonstrated in Table 2 that the ASr-UKF algorithm still has better performance than the other filters.

\subsubsection{Noise process with unknown time-varying statistics}

This section reports the simulations when noise processes affect the manipulator. In this case, the covariance of noises are defined as follows:

- for $t<5 \mathrm{~s}, Q=10^{-5} I_{6 \times 6}, R=10^{-5} I_{6 \times 6}$;

- for $5 \mathrm{~s} \leq \mathrm{t} \leq 15 \mathrm{~s}: Q=10^{-4} I_{6 \times 6}, R=10^{-7} I_{6 \times 6}$;

- for $t>15 \mathrm{~s}, Q=10^{-5} I_{6 \times 6}, R=10^{-5} I_{6 \times 6}$.

Despite these varying conditions, the new ASr-UKF filter has to estimate the states of the robotic manipulator. The initial values for the estimation covariances are set as $\hat{Q}=10^{-20} I_{6 \times 6}$ and $\hat{R}=10^{-20} I_{6 \times 6}$. The starting point of the estimated system and actual system is initialized as $X_{0}=[1,0.5,1,2,-1,-2]^{T}$ and $\hat{X}_{0}=[0,0,0,0,0,0]^{T}$, respectively. The simulation results for the links of the robot are reported in Fig. 4 . These results are compared with those obtained with Sr-UKF, UKF, and EKF. The estimation results and its error for the velocity of robot's links are summarized in Fig. 5 . As can be seen in Figs. 4 and 5 , the conventional filters do not have adaptation

Table 3: Robustness evaluation of the robotic manipulator affected by noises with constant statistics and different initial conditions by the percent normalized mean square error criteria

\begin{tabular}{|c|c|c|c|c|c|c|}
\hline Variable & $\theta_{1}$ & $\theta_{2}$ & $\theta_{3}$ & $\dot{\theta}_{1}$ & $\dot{\theta}_{2}$ & $\dot{\theta}_{3}$ \\
\hline$\hat{X}_{0}=[0,0,0,0,0,0]^{T}$ & 0.73 & 0.13 & 0.07 & 0.82 & 0.36 & 0.63 \\
\hline$\hat{X}_{0}=[1,0,1,2,-1,-2]^{T}$ & 0.22 & 0.13 & 0.02 & 0.12 & 0.11 & 0.09 \\
\hline$\hat{X}_{0}=[1,1,1,3,-1,-3]^{T}$ & 0.21 & 0.13 & 0.03 & 0.42 & 0.10 & 0.32 \\
\hline$\hat{X}_{0}=[4.3,2.62,7,6.65,3.5,8.75]^{T}$ & 2.49 & 0.49 & 0.41 & 2.49 & 1.82 & 4.81 \\
\hline
\end{tabular}



(a) First link's position
(c) Second link's position
(e) Third link's position

(b) Estimation error of the first link's position

(d) Estimation error of the second link's position

(f) Estimation error of third link's position

Figure 4: Comparison of estimated states and estimated errors of the robotic manipulator: components of the robot's position affected by noises with time-varying statistics (True: —, ASr-UKF: —, Sr-UKF: —, UKF: - , EKF: —).

Table 4: Percent normalized mean square error of estimation of the robotic manipulator affected by noises with time-varying statistics

\begin{tabular}{|c|c|c|c|c|c|c|}
\hline Vethod & $\theta_{1}$ & $\theta_{2}$ & $\theta_{3}$ & $\dot{\theta}_{1}$ & $\dot{\theta}_{2}$ & $\dot{\theta}_{3}$ \\
\hline ASr-UKF & 0.47 & 0.14 & 0.14 & 0.66 & 0.32 & 0.54 \\
\hline Sr-UKF & 60.62 & 57.00 & 43.45 & 4.70 & 7.80 & 8.90 \\
\hline UKF & 9.50 & 2.40 & 0.70 & 3.48 & 3.75 & 5.01 \\
\hline AUKF & 0.65 & 0.27 & 0.21 & 0.81 & 0.51 & 0.68 \\
\hline EKF & 18.92 & 3.94 & 8.54 & 4.71 & 7.91 & 9.53 \\
\hline AEKF & 0.83 & 0.29 & 0.27 & 1.02 & 0.69 & 0.70 \\
\hline
\end{tabular}

capabilities and they are not able to provide effective estimates of the monitored states. To provide more effective comparisons, the percent normalized mean square errors of the estimations for all methods are presented in Table 4 The results show that the presented the ASr-UKF can provide more accurate estimations than traditional filters.
(a) First link's velocity
(b) Estimation error of the first link's velocity
(c) Second link's velocity
(d) Estimation error of the second link's velocity
(e) Third link's velocity
(f) Estimation error of third link's velocity

Figure 5: Comparison of estimated states and estimated errors of the robotic manipulator: components of the robot's velocity affected by noises with time-varying statistics (True: —, ASr-UKF: —, Sr-UKF: —, UKF: - , EKF: —).

To evaluate the robustness of the proposed ASr-UKF with respect to the estimated initial values, the percent normalized mean square-root is listed in Table 5 for different initial values. These values are chosen randomly. It is obvious that the proposed ASr-UKF is robust with respect to the changes in initial conditions.

\subsection{Servo-hydraulic system}

We now consider a second case study represented by a servo-hydraulic process. The schematic diagram of this system is sketched in Fig. 6. The mathematical relation describing the behaviour of the system has the following form:

$$
M \ddot{x}_{P}=-b \dot{x}_{P}+A_{1} p_{1}-A_{2} p_{2}-F_{e}
$$

where $x_{P}$ represents the piston position. The pressure and the area of the cylinder are represented by the parameters $p_{1}$, $P_{2}$ and $A_{1}, A_{2}$, respectively. The parameters $M, F_{e}$, and $b$ represent the load mass, external force, and friction, 
Table 5: Robustness evaluation of the robotic manipulator affected by noises with time-varying statistics and different initial conditions by the percent normalized mean square error criteria

\begin{tabular}{|c|c|c|c|c|c|c|}
\hline${ }_{\mathrm{ASr}-\mathrm{UKF}}$ Variable & $\theta_{1}$ & $\theta_{2}$ & $\theta_{3}$ & $\dot{\theta}_{1}$ & $\dot{\theta}_{2}$ & $\dot{\theta}_{3}$ \\
\hline$\hat{X}_{0}=[0,0,0,0,0,0]^{T}$ & 0.47 & 0.14 & 0.14 & 0.68 & 0.36 & 0.57 \\
\hline$\hat{X}_{0}=[2,1,3,3,2,4]^{T}$ & 0.51 & 0.15 & 0.24 & 0.40 & 0.90 & 1.69 \\
\hline$\hat{X}_{0}=[2,1,4,4,2,5]^{T}$ & 0.50 & 0.14 & 0.34 & 0.86 & 1.14 & 2.07 \\
\hline$\hat{X}_{0}=[1,1,2,2,1,2]^{T}$ & 0.29 & 0.14 & 0.13 & 0.23 & 0.60 & 1.13 \\
\hline
\end{tabular}

respectively [38. To determine the position of the piston, Eq. 20] can be used to derive the pressure on the two sides of the cylinder. By applying the basic hydraulic laws [39], the pressures of the two sides of the cylinder are defined by the following relations:

$$
\begin{aligned}
& \dot{P}_{1}=\frac{\beta_{e}}{V_{1}}\left(Q_{1}-A_{1} \dot{x}_{P}+Q_{I}-Q_{E 1}\right) \\
& \dot{P}_{2}=\frac{\beta_{e}}{V_{2}}\left(Q_{2}-A_{2} \dot{x}_{P}-Q_{I}-Q_{E 2}\right)
\end{aligned}
$$

where the fluid flows of each side of the cylinder are represented by $Q_{1}$, and $Q_{2}$. The parameters $\beta_{e}, V_{1}$, and $V_{2}$ represent the bulk modulus and the volume of each side of the cylinder, respectively. The internal leakage and external leakage of each side are given by $Q_{I}, Q_{E 1}$, and $Q_{E 2}$, respectively. Different flows in the hydraulic circuit can be described by the following relations:

$$
\begin{aligned}
& Q_{1}= \begin{cases}C_{s} u \sqrt{p_{s}-p_{1}} & u \geq 0 \\
C_{s} u \sqrt{p_{1}-p_{a}} & u<0\end{cases} \\
& Q_{2}= \begin{cases}C_{s} u \sqrt{p_{2}-p_{a}} & u \geq 0 \\
C_{s} u \sqrt{p_{s}-p_{2}} & u<0\end{cases} \\
& Q_{I}=K_{i}\left(p_{2}-p_{1}\right) \\
& Q_{E 1}=K_{E 1}\left(p_{1}-p_{a}\right) \\
& Q_{E 2}=K_{E 2}\left(p_{2}-p_{a}\right)
\end{aligned}
$$

The relations in 22 need the volume on different sides of the cylinder. These values can be calculated using the following relations:

$$
\begin{aligned}
& V_{1}=A_{1} x_{P}+v_{01} \\
& V_{2}=A_{2}\left(L-x_{P}\right)+v_{02}
\end{aligned}
$$

where the dead volume of each side is indicated with $v_{0 i}$. The parameter $L$ represents the maximum value of the piston position.

As for the first case study, three scenarios are considered in the simulation of the hydraulic system. The results of the simulation are presented in the following. 


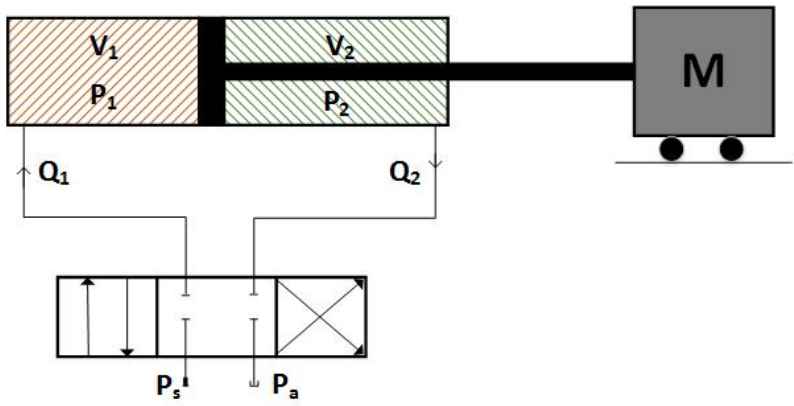

Figure 6: The schematic of servo-hydraulic system

Table 6: Percent normalized mean square error of estimation of a servo-hydraulic system affected by noises with known constant statistics

\begin{tabular}{|c|c|c|c|c|}
\hline Method & $X_{p}$ & $\dot{X}_{p}$ & $P_{1}$ & $P_{2}$ \\
\hline ASr-UKF & 0.496 & 0.616 & 2.518 & 1.271 \\
\hline Sr-UKF & 0.493 & 0.616 & 2.511 & 1.271 \\
\hline UKF & 0.475 & 0.617 & 2.518 & 1.371 \\
\hline AUKF & 0.489 & 0.616 & 2.510 & 1.281 \\
\hline EKF & 2.269 & 1.501 & 2.621 & 1.518 \\
\hline AEKF & 0.782 & 0.947 & 2.593 & 1.372 \\
\hline
\end{tabular}

\subsubsection{Noise with known constant statistics}

The performance of the proposed ASr-UKF is studied when the statistics of the noise are known. To this end, the true values of the noises are considered as $Q=10^{-6} I_{6 \times 6}$ and $R=10^{-4} I_{6 \times 6}$. Fig. 7 represents the performance of the ASr-UKF in comparison to other filters. To have a better comparision metric, the percent normalized mean square error of this simulation is shown in Tab. 6. The results indicate the acceptable performance of the ASr-UKF algorithm. The other filters have almost the same results.
(a) Estimation of $X_{p}$
(b) Estimation error of $X_{p}$
(c) Estimation of $\dot{X}_{p}$
(d) Estimation error of $\dot{X}_{p}$
(e) Estimation of $P_{1}$
(f) Estimation error of $P_{1}$
(g) Estimation of $P_{2}$
(h) Estimation error of $P_{2}$

Figure 7: Comparison of estimated states and estimated errors of the servo-hydraulic system: affected by noises with known constant statistics (True: $\longrightarrow$, ASr-UKF: —, Sr-UKF: —, UKF: - , EKF: 一).

\subsubsection{Noise with unknown constant statistics}

The first case assumes that two zero-mean noises affect the process and measurement of the servo-hydraulic system. The covariance of the real noises is set as $Q=10^{-3} I_{6 \times 6}$ and $R=10^{-3} I_{6 \times 6}$, which represent the covariance of process and measurement noises, respectively. As mentioned previously, one of the advantages of the method 
Table 7: Percent normalized mean square error of estimation of a servo-hydraulic system affected by noises with constant statistics

\begin{tabular}{|c|c|c|c|c|}
\hline Method & $X_{p}$ & $\dot{X}_{p}$ & $P_{1}$ & $P_{2}$ \\
\hline ASr-UKF & 1.80 & 0.78 & 2.49 & 2.52 \\
\hline Sr-UKF & 9.11 & 6.72 & 15.01 & 16.70 \\
\hline UKF & 23.94 & 14.55 & 24.47 & 28.07 \\
\hline AUKF & 1.71 & 0.78 & 2.54 & 2.52 \\
\hline EKF & 29.43 & 21.11 & 31.96 & 37.24 \\
\hline AEKF & 2.12 & 0.76 & 2.61 & 2.51 \\
\hline
\end{tabular}

proposed in this work is its independence from a priori knowledge about the noise. Regarding this fact, the initial values of the estimated covariance are set to $\hat{Q}=10^{-20} I_{6 \times 6}$ and $\hat{R}=10^{-20} I_{6 \times 6}$. The estimated system and actual plant start from $\hat{X}_{0}=[0,0,0,0]^{T}$ and $X_{0}=[0.18,0.0002,2 e 6,2 e 6]^{T}$, respectively. After these assumptions, the results of the simulation are summarized as Fig. 8 . As in the previous simulations, the proposed method is compared with Sr-UKF, UKF, EKF, AUKF, and AEKF. The Fig. 8 shows that the proposed ASr-UKF provides more accurate estimation when compared with the other filters. To have a more precise view, the percent normalized mean square error of each method is reported in Table 7
(a) Estimation of $X_{p}$
(b) Estimation error of $X_{p}$
(c) Estimation of $\dot{X}_{p}$
(d) Estimation error of $\dot{X}_{p}$
(e) Estimation of $P_{1}$
(f) Estimation error of $P_{1}$
(g) Estimation of $P_{2}$
(h) Estimation error of $P_{2}$

Figure 8: Comparison of estimated states and estimated errors of the servo-hydraulic system: affected by noises with constant statistics (True: —, ASr-UKF: —, Sr-UKF: —, UKF: —, EKF: —).

\subsubsection{Noise with unknown time-varying statistics}

This section considers the case where noise with time-varying statistics affects the servo-hydraulic system. The covariance of the noise process changes according to the following rules:

- for $t<4 \mathrm{~s}, Q=10^{-7} I_{6 \times 6}, R=10^{-5} I_{6 \times 6}$;

- for $4 \mathrm{~s} \leq \mathrm{t} \leq 16 \mathrm{~s}, Q=10^{-5} I_{6 \times 6}, R=10^{-3} I_{6 \times 6}$;

- for t $>16 \mathrm{~s}, Q=10^{-7} I_{6 \times 6}, R=10^{-5} I_{6 \times 6}$.

Even if the power of the noise processes, which affect states and measurement of the hydraulic system, changes over time, the filter is able to estimate the states of the plant by updating the estimated covariances. The initial values of the covariance matrices are set to $\hat{Q}=10^{-20} I_{6 \times 6}$ and $\hat{R}=10^{-20} I_{6 \times 6}$. The initial conditions are set as $X_{0}=[0.2,0.0002,2.5 e 6,2.5 e 6]^{T}$ and $\hat{X}_{0}=[0,0,0,0]^{T}$. Based on these assumptions, the simulation results are shown 
in Fig. 9. As for this case, because the hydraulic system has severe nonlinearity, the conventional EKF was not able to estimate the states and became "not a number" (NaN). Therefore, the EKF plot is removed from Fig. 9
(a) Estimation of $X_{p}$
(b) Estimation error of $X_{p}$
(c) Estimation of $\dot{X}_{p}$
(d) Estimation error of $\dot{X}_{p}$
(e) Estimation of $P_{1}$
(f) Estimation error of $P_{1}$
(g) Estimation of $P_{2}$
(h) Estimation error of $P_{2}$

Figure 9: Comparison of estimated states and estimated errors of the servo-hydraulic system: affected by noises with time-varying statistics (True: —, ASr-UKF: —, Sr-UKF: —, UKF: $—$ ).

The percent normalized mean square error of each state of the system is summarized in Table 8 .

Table 8: Percent normalized mean square error of estimation of a servo-hydraulic system affected by noises with time-varying statistics

\begin{tabular}{|c|c|c|c|c|}
\hline Method & $X_{p}$ & $\dot{X}_{p}$ & $P_{1}$ & $P_{2}$ \\
\hline ASr-UKF & 0.80 & 1.29 & 1.00 & 0.17 \\
\hline Sr-UKF & 46.10 & 42.77 & 26.79 & 11.14 \\
\hline UKF & 46.12 & 19.17 & 12.81 & 14.07 \\
\hline AUKF & 0.82 & 1.38 & 1.14 & 0.16 \\
\hline EKF & $N a N^{*}$ & $N a N$ & $N a N$ & $N a N$ \\
\hline AEKF & 0.91 & 1.34 & 1.13 & 0.17 \\
\hline
\end{tabular}

* NaN is an abbreviation for "not a number."

\subsubsection{Experimental results}

To test the proficiency of the proposed method, it is applied on a real-world practical system. A servo-hydraulic actuator is chosen as a practical case study. The system is situated in the Laboratory of Intelligent Machines at LUT University. The structure of the system is shown in Fig. 10. The overall procedure of data collection from practical set can be explained as follows:

- The supply pressure is controlled by a constant pressure pump driven by an inverter

- Control signal is produced by High speed PC

- dSpace module convert the produced control to analog format and sends it to the system

- New data in servo-hydraulic system, which is a consequence of new control signal, are collected using sensors

- dSpace module converts collected analog measurements into diginal signal

- High speed PC collects measured data and produces new control signal 
Table 9: Percent normalized mean square error of estimation of a servo-hydraulic system in a practical application

\begin{tabular}{|c|c|c|c|c|}
\hline \multicolumn{2}{|c|}{ Initial covariances values } & \multicolumn{3}{|c|}{ Hydraulic system states } \\
\hline$\hat{Q}_{0}$ & $\hat{R}_{0}$ & $x_{p}$ & $P_{1}$ & $P_{2}$ \\
\hline $10^{-10}$ & $10^{-10}$ & 1.36 & 2.94 & 1.60 \\
\hline $10^{-8}$ & $10^{-8}$ & 2.11 & 1.85 & 0.97 \\
\hline $10^{-2}$ & $10^{-2}$ & 1.84 & 4.28 & 1.98 \\
\hline $10^{-11}$ & $10^{-18}$ & 3.93 & 5.33 & 3.12 \\
\hline
\end{tabular}

The proposed method is applied on the collected data from the practical system, and the results can be found in Fig. 11. For the estimation, the position of the piston is considered as the output of the system and other states are estimated regarding this output. Owing to the practical instruments, it was possible to collect data for three states of the system, $x_{p}, P_{1}$, and $P_{2}$. The collected data can be found in "http://dx.doi.org/10.17632/cg6p86pg8j.1'. Percent normalized mean square error of estimation for each state of the system is $x_{p}=1.36, P_{1}=9.80$, and $P_{2}=6.90$. Comparison between collected data and estimated values for all three states proves that the proposed method can be considered as a strong and reliable approach for estimation of the states of practical systems. In order to test the validity of the proposed method in different conditions with different reference input signals, some experimental tests were done which their percent normalized MSE are sketched in Tab. 9. As it can be seen in the table, the proposed method is capable to preform with acceptable error. The presented results guarantee the universality of the filtering algorithm. The experimental data set, which have been used for the validity check, are available in "http://dx.doi.org/10.17632/9nb3vbbtjw.1'.

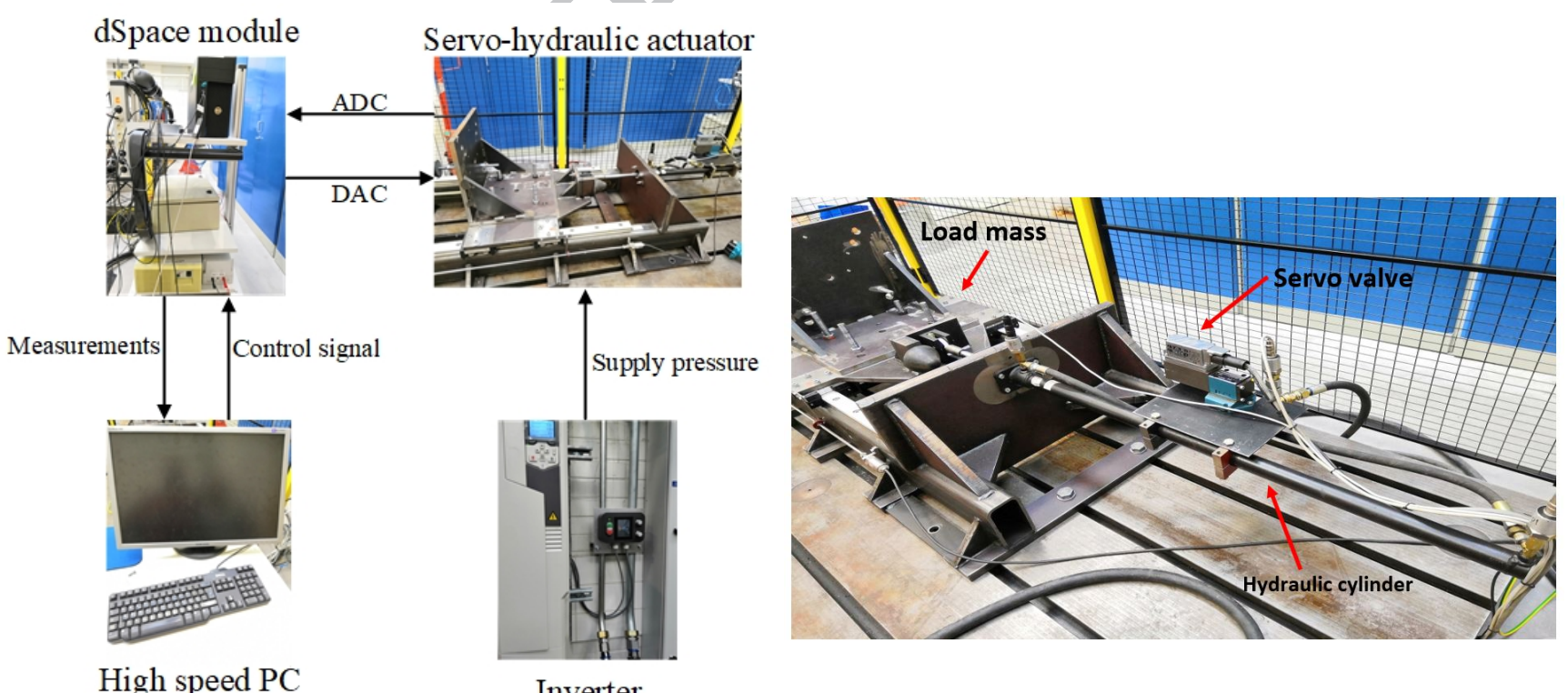

(a) Hydraulic test setup

(b) Servo-hydraulic actuator

Figure 10: Practical servo-hydraulic actuator system in the Laboratory of intelligent Machines at LUT University. 

(a) Estimation of $x_{p}$
(b) Estimation error of $x_{p}$
(c) Estimation of $P_{1}$
(d) Estimation error of $P_{1}$
(e) Estimation of $P_{2}$
(f) Estimation error of $P_{2}$

Figure 11: State estimation of the experimental hydraulic actuator: affected by noises with unknown statistics (Collected data: — ASr-UKF: - )

\subsection{Hydraulic actuator leakage fault detection}

- the generated residuals are compared with the detection threshold;

- a fault occurrence is detected when the residual signals exceed the fixed threshold.

The residual generator scheme and the residual evaluation phase are summarized in Fig. 12 , Based on the abovementioned steps, it is necessary to modify the model of 21] to include the fault description. As remarked previously, internal and external leakages for the first and second side of the cylinder are described by $Q_{I}, Q_{E 1}$, and $Q_{E 2}$, 
respectively. According to this description, the model of the system is modified in the following form:

$$
\begin{aligned}
& \dot{P}_{1}=\frac{\beta_{e}}{V_{1}}\left(Q_{1}-A_{1} \dot{x}_{P}\right) \\
& \dot{P}_{2}=\frac{\beta_{e}}{V_{2}}\left(Q_{2}-A_{2} \dot{x}_{P}\right)
\end{aligned}
$$

To have a clearer view of the proposed fault detection approach, the achieved results are presented in the following.

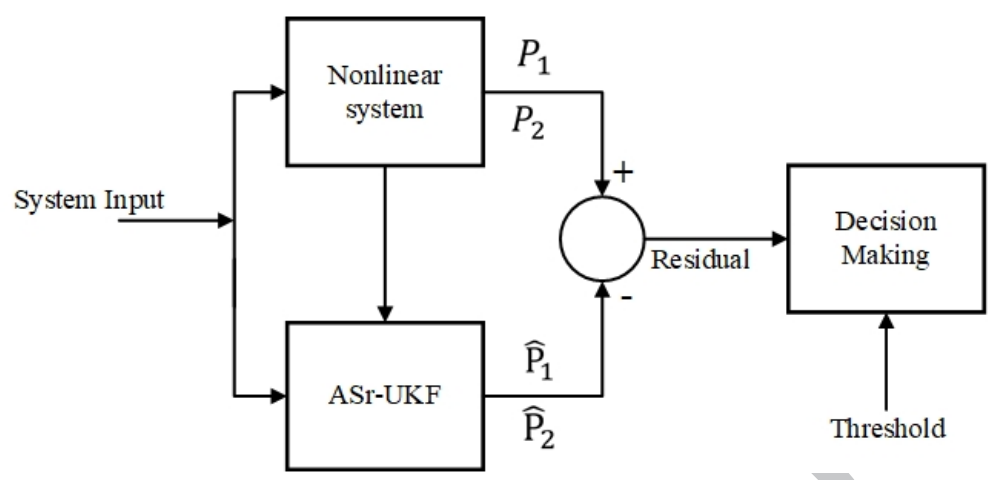

Figure 12: Diagram of the residual generation and evaluation modules.

\subsubsection{Noise with constant statistics}

As a first test, the process and measurement noises are considered to be constant over time. The actual noise statis-

tics that affect the system are assumed to be zero mean with process covariance of $Q=10^{-5} I_{6 \times 6}$ and measurement covariance of $R=10^{-4} I_{6 \times 6}$. The initial values of the estimated covariances are set to $\hat{Q}=10^{-10} I_{6 \times 6}$ and $\hat{R}=10^{-10} I_{6 \times 6}$ and in the same way, the initial condition point of the system and its estimation is $X_{0}=[0.2,0.0002,2.5 e 6,5 e 6]^{T}$ and $\hat{X}_{0}=[0,0,0,0]^{T}$, respectively. It has also been assumed that the threshold is $200(\mathrm{~Pa})$ for $P_{1}$ and $P_{2}$. To simulate the leakage fault, described by (24), it is assumed that the system is working in a fault-free condition from $t=0$ to $10 \mathrm{~s}$, which is when the fault occurs and lasts to the end of the simulation.

The results of the simulation are summarized in Fig. 13. It can be seen from Fig. 13a that the proposed filter is capable of following the true values of $P_{1}$ after $t=10 \mathrm{~s}$ with high accuracy, even though the noise statistics are unknown. By analysing Fig. 13b it is obvious that a fault has occurred for $t>10 \mathrm{~s}$, as the residual signal exceeds the threshold fixed in fault-free conditions. Therefore, the threshold $200(\mathrm{~Pa})$ allows the considered fault case to be detected properly. The same results hold for the signal $P_{2}$, which are shown in Figs. $13 \mathrm{c}$ and $13 \mathrm{~d}$.
(a) Estimation of $P_{1}$
(b) Residual signal of $P_{1}$
(c) Estimation of $P_{2}$
(d) Residual signal of $P_{2}$

Figure 13: Generated residual signals for leakage fault detection of hydraulic actuator: affected by noises with constant statistics (True: —, ASr-UKF: —, Sr-UKF: —).

\subsubsection{Noise with time-varying statistics}

To further study the performance of ASr-UKF for fault detection, the time-varying noise distribution is considered in this section. The changes of noise covariance variations are defined as: 
- for $t<4 \mathrm{~s}, Q=10^{-5} I_{6 \times 6}, R=10^{-4} I_{6 \times 6}$;

- for $4 \mathrm{~s} \leq \mathrm{t} \leq 16 \mathrm{~s}: Q=10^{-4} I_{6 \times 6}, R=10^{-3} I_{6 \times 6}$;

- for $t>16 \mathrm{~s}, Q=10^{-5} I_{6 \times 6}, R=10^{-4} I_{6 \times 6}$.

The initial conditions are set as $X_{0}=[0.2,0.0002,2.5 e 6,5 e 6]^{T}$ and $\hat{X}_{0}=[0,0,0,0]^{T}$. The fault is assumed to occur at $t=15 \mathrm{~s}$ for both pressures. The threshold is set to $50(\mathrm{~Pa})$ for both $P_{1}$ and $P_{2}$.

The simulated results are shown in Fig. 14. Although the process and measurement noise processes vary over time, the accuracy of the ASr-UKF allows for accurate fault detection capabilities. In fact, Figs. 14b and 14d show that the residual signals are different from zero for $t>15 \mathrm{~s}$. The threshold fixed at $50(\mathrm{~Pa})$ allows the fault for $t \geq 15$ $\mathrm{s}$ to be detected.
(a) Estimation of $P_{1}$
(b) Residual signal of $P_{1}$
(c) Estimation of $P_{2}$
(d) Residual signal of $P_{2}$

Figure 14: Generated residual signals for leakage fault detection of hydraulic actuator: affected by noises with time-varying statistics (True: —, ASr-UKF: —, Sr-UKF: —).

\section{Conclusion}

The aim of this study was to introduce a new version of square-root unscented Kalman filter. The presented filter was designed so that it can adapt itself over time. Whilst other traditional estimation filters need a priori knowledge about the noise covariance that affects the system, the presented filter does not require such information and it is capable to estimate the noise statistics of the process and measurement noises in an adaptive manner. The filter was derived by means of basic probabilistic equations, which guaranteed its stability. The performances of this filter were investigated by considering two applications of state estimation and fault detection. In particular, the presented filter was applied to estimate the states of two systems, a robotic manipulator and a servo-hydraulic actuator. The achieved results were compared with well-established estimation methods relying on Kalman filters. The simulations and experimental application highlighted that the proposed filter provides accurate estimations when the other filters fail in the presence of both unknown fixed and varying noise statistics. Finally, the ability of the presented filter to detect a leakage fault in a hydraulic system has also been tested. The results proved that the filter can also be exploited as a reliable instrument for fault detection. Further studies will verify the reliability and robustness features of the developed tool, when used also for fault-tolerant control applications, as the accuracy of the estimated signals is very important.

[1] D. Ding, Q.-L. Han, Z. Wang, X. Ge, A survey on model-based distributed control and filtering for industrial cyber-physical systems, IEEE Transactions on Industrial Informatics.

[2] W. Chen, D. Ding, H. Dong, G. Wei, Distributed resilient filtering for power systems subject to denial-of-service attacks, IEEE Transactions on Systems, Man, and Cybernetics: Systems. 
[3] H. Bae, J.-H. Oh, Biped robot state estimation using compliant inverted pendulum model, Robotics and Autonomous Systems 108 (2018) 38-50.

[4] R. M. Asl, F. Hashemzadeh, M. A. Badamchizadeh, A new adaptive neural network based observer for robotic manipulators, in: 2015 3rd RSI international conference on robotics and mechatronics (ICROM), IEEE, 2015, pp. 663-668.

[5] W. Gu, G. Lou, W. Tan, X. Yuan, A nonlinear state estimator-based decentralized secondary voltage control scheme for autonomous microgrids, IEEE Transactions on Power Systems 32 (6) (2017) 4794-4804.

[6] Y. Nakahira, Y. Mo, Attack-resilient $h_{2}, \mathrm{H}_{\infty}$, and $\ell_{1}$ state estimator, IEEE Transactions on Automatic Control 63 (12) (2018) 4353-4360.

[7] F. Gustafsson, Particle filter theory and practice with positioning applications, IEEE Aerospace and Electronic Systems Magazine 25 (7) (2010) 53-82.

[8] D. Simon, Kalman filtering with state constraints: a survey of linear and nonlinear algorithms, IET Control Theory \& Applications 4 (8) (2010) 1303-1318.

[9] F. Gustafsson, F. Gunnarsson, N. Bergman, U. Forssell, J. Jansson, R. Karlsson, P.-J. Nordlund, Particle filters for positioning, navigation, and tracking, IEEE Transactions on signal processing 50 (2) (2002) 425-437.

[10] C. K. Chui, G. Chen, et al., Kalman filtering, Springer, 2017.

[11] S. Safari, F. Shabani, D. Simon, Multirate multisensor data fusion for linear systems using kalman filters and a neural network, Aerospace Science and Technology 39 (2014) 465-471.

[12] X. Yin, L. Zhang, Z. Ning, D. Tian, A. Alsaedi, B. Ahmad, State estimation via markov switching-channel network and application to suspension systems, IET Control Theory \& Applications 11 (3) (2016) 411-419.

[13] L. Li, M. Yang, C. Wang, B. Wang, Rigid point set registration based on cubature kalman filter and its application in intelligent vehicles, IEEE Transactions on Intelligent Transportation Systems 19 (6) (2018) 17541765.

[14] A. K. Sivathanu, S. Subramanian, Extended kalman filter for fouling detection in thermal power plant reheater, Control Engineering Practice 73 (2018) 91-99.

[15] L. Guo, Y. Ding, Z. Wang, G. Xu, B. Wu, A dynamic load estimation method for nonlinear structures with unscented kalman filter, Mechanical Systems and Signal Processing 101 (2018) 254-273.

[16] K. Jiang, H. Zhang, J. Lin, Nh _\{3\} coverage ratio estimation of diesel-engine scr systems by a dual time-scale extended kalman filter, IEEE Transactions on Vehicular Technology 67 (4) (2017) 3625-3629.

[17] R. Mohammadi Asl, Y. Shabbouei Hagh, R. Palm, Robust control by adaptive non-singular terminal sliding mode, Engineering Applications of Artificial Intelligence 59 (2017) 205 - 217. 
[18] K. Jiang, F. Yan, H. Zhang, Hydrothermal aging factor estimation for two-cell diesel-engine scr systems via a dual time-scale unscented kalman filter, IEEE Transactions on Industrial Electronics.

[19] Y. Liu, X. Fan, C. Lv, J. Wu, L. Li, D. Ding, An innovative information fusion method with adaptive kalman filter for integrated ins/gps navigation of autonomous vehicles, Mechanical Systems and Signal Processing 100 (2018) 605-616.

[20] R. M. Asl, H. Handroos, New weighted adaptive unscented kalman filter for estimation of hydraulic systems, in: 2018 Global Fluid Power Society PhD Symposium (GFPS), IEEE, 2018, pp. 1-6.

[21] Y. Lin, K. Liu, X. Wang, An adaptive square-root unscented kalman filter for underwater vehicle navigation, in: 2014 IEEE International Conference on Mechatronics and Automation, IEEE, 2014, pp. 717-722.

[22] R. Van Der Merwe, E. A. Wan, The square-root unscented kalman filter for state and parameter-estimation, in: Acoustics, Speech, and Signal Processing, 2001. Proceedings.(ICASSP'01). 2001 IEEE International Conference on, Vol. 6, IEEE, 2001, pp. 3461-3464.

[23] J. Guo, H. Zhang, Y. Sun, R. Bie, Square-root unscented kalman filtering-based localization and tracking in the internet of things, Personal and ubiquitous computing 18 (4) (2014) 987-996.

[24] S. X. Ding, Model-based fault diagnosis techniques: design schemes, algorithms, and tools, Springer Science \& Business Media, 2008.

[25] J. Chen, R. J. Patton, Robust model-based fault diagnosis for dynamic systems, Vol. 3, Springer Science \& Business Media, 2012.

[26] Q. Zhang, Adaptive kalman filter for actuator fault diagnosis, Automatica 93 (2018) 333-342.

[27] V. R. Palleti, Y. C. Tan, L. Samavedham, A mechanistic fault detection and isolation approach using kalman filter to improve the security of cyber physical systems, Journal of Process Control 68 (2018) 160-170.

[28] K. Lee, J. Cha, S. Ko, S.-Y. Park, E. Jung, Fault detection and diagnosis algorithms for an open-cycle liquid propellant rocket engine using the kalman filter and fault factor methods, Acta Astronautica.

[29] L. An, N. Sepehri, Leakage fault detection in hydraulic actuators subject to unknown external loading, International Journal of Fluid Power 9 (2) (2008) 15-25.

[30] E. A. Wan, R. Van Der Merwe, The unscented kalman filter, Kalman filtering and neural networks (2001) $221-280$.

[31] L. Zhao, J. Wang, T. Yu, H. Jian, T. Liu, Design of adaptive robust square-root cubature kalman filter with noise statistic estimator, Applied Mathematics and Computation 256 (2015) 352 - 367.

[32] M. Xing, Q. Hongzhuan, An adaptive ukf algorithm and its application for satellite attitude determination, in: Consumer Electronics, Communications and Networks (CECNet), 2011 International Conference on, IEEE, 2011, pp. 78-81. 
[33] C. Hoffmann, S. M. Hashemi, H. S. Abbas, H. Werner, Benchmark problemnonlinear control of a 3-dof robotic manipulator, in: Decision and Control (CDC), 2013 IEEE 52nd Annual Conference on, IEEE, 2013, pp. 55345539.

[34] E. A. Wan, R. Van Der Merwe, The unscented kalman filter for nonlinear estimation, in: Proceedings of the IEEE 2000 Adaptive Systems for Signal Processing, Communications, and Control Symposium (Cat. No. 00EX373), Ieee, 2000, pp. 153-158.

[35] M. Gautier, P. Poignet, Extended kalman filtering and weighted least squares dynamic identification of robot, Control Engineering Practice 9 (12) (2001) 1361-1372.

[36] Y. Shabbouei Hagh, R. Mohammadi Asl, V. Cocquempot, A hybrid robust fault tolerant control based on adaptive joint unscented kalman filter, ISA Transactions 66 (2017) $262-274$.

[37] R. M. Asl, Y. S. Hagh, H. Handroos, Adaptive extended kalman filter designing based on non-singular fast terminal sliding mode control for robotic manipulators, in: 2017 IEEE International Conference on Mechatronics and Automation (ICMA), IEEE, 2017, pp. 1670-1675.

[38] H. Yousefi, H. Handroos, J. K. Mattila, Application of fuzzy gain-scheduling in position control of a servo hydraulic system with a flexible load, International Journal of Fluid Power 8 (2) (2007) 25-35.

[39] M. Jelali, A. Kroll, Hydraulic servo-systems: modelling, identification and control, Springer Science \& Business Media, 2012.

[40] Y. Zhang, J. Jiang, Bibliographical review on reconfigurable fault-tolerant control systems, Annual reviews in control 32 (2) (2008) 229-252.

[41] R. Isermann, Fault-diagnosis systems: an introduction from fault detection to fault tolerance, Springer Science \& Business Media, 2006. 